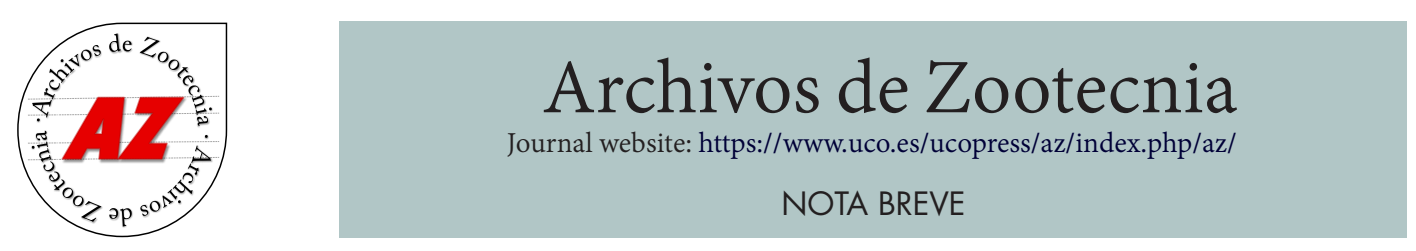

\title{
Parâmetros genéticos para características de crescimento e reprodutivas em bovinos Nelore no Brasil
}

Barbosa, A.C.B. ${ }^{@}{ }^{1}$ Carneiro, P.L.S. ${ }^{2}$; Rezende, M.P.G. ${ }^{2}$; Ramos, I.O. ${ }^{2}$; Martins Filho, R. ${ }^{3}$ e Malhado, C.H.M. ${ }^{2}$

1 Universidade Federal da Bahia. Salvador. Bahia. Brasil.

${ }^{2}$ Universidade Estadual do Sudoeste da Bahia. Jequié. Bahia. Brasil.

${ }^{3}$ Universidade Federal do Cariri. Campus de Juazeiro do Norte. Ceará. Brasil.

PALAVRAS-CHAVE ADICIONAIS

Ganho genético.

Tendência genética.

Tendência fenotípica.

\begin{abstract}
RESUMO
Conhecer o progresso genético e fenotípico de uma população é importante para se avaliar o resultado dos programas de seleção adotados e como elementos norteadores de ações futuras. Assim, objetivou-se estimar parâmetros genéticos, tendências genéticas e fenotípicas dos pesos aos 205 (P205), 365 (P365) e 550 (P550) dias de idade, idade ao primeiro parto (IPP), circunferência escrotal (CE) e intervalos de partos (IDP) de 175.231 bovinos Nelore, nascidos no período de 1967 a 2011 na região Nordeste do Brasil. Os componentes de (co) variância e valores genéticos foram obtidos por meio da metodologia da máxima verossimilhança restrita. As estimativas dos coeficientes de herdabilidades para as características de crescimento foram de baixa a moderada magnitude. A seleção direta proporcionará maiores ganhos genéticos para P550. Para as características reprodutivas, a estimativa de herdabilidade foi baixa para IPP e próxima a zero para IDP. Contudo, para a CE a herdabilidade foi moderada, indicando seu uso como critério de seleção. As tendências genéticas diretas para P205, P365, P550 e IPP, indicam pequenos ganhos genéticos ao longo dos anos. Os progressos fenotípicos evidenciam que as características estão melhorando com o decorrer dos anos, principalmente em decorrência da melhoria ambiental.
\end{abstract}

Genetics parameters for growth and reproductive traits in Nellore Cattle from Brazil

\section{SUMMARY}

To know the genetic and phenotypic progress of a population is important to evaluate the outcome of the adopted selection programmes and as guiding elements of future actions. The objective was to estimate genetic parameters, genetic and phenotypic trends of weights at 205 (W205) 365 (W365) 550 (W550) days of age, age at first calving (AFC), scrotal circumference (SC) and calving interval (CI) of 175.231 Nellore, born in the period 1967-2011 in the Northeast region of Brazil. The components of $(\mathrm{co})$ variance and breeding values were obtained by restricted maximum likelihood methodology. Estimates of heritability coefficients for growth traits were of a low to moderate magnitude. Direct selection will provide greater genetic gains for W550. For reproductive traits, the heritability estimate was low for ACF and close to zero for $\mathrm{Cl}$. However, for the SC heritability was moderate, indicating its use as selection criteria. Direct genetic trends for W205, W365, W550 and ACF indicate small genetic gains over the years. Phenotypic progress shows that the features are improving with the years, mainly because of environmental improvements.

INFORMATION

Cronología del artículo.

Recibido/Received: 27.07.2016

Aceptado/Accepted: 27.02.2017

On-line: 15.07.2017

Correspondencia a los autores/Contact e-mail:

linhabio@gmail.com

\section{INTRODUÇÃO}

A performance das características de crescimento e reprodutivas na bovinocultura de corte sofrem influências ambientais (Fialho et al., 2015; Oliveira et al., 2015), podendo ser determinante na lucratividade da produção (Laureano et al., 2011).
Essa situação é comum no nordeste brasileiro, onde os bovinos de corte são criados a pasto (Biffani et al., 1999), rebanhos compostos, basicamente, por animais zebuínos, principalmente a raça Nelore, devido a sua grande adaptação às condições edafoclimáticas desta região. Entretanto, os índices produtivos destes animais são pequenos e 
têm como um dos motivos a falta de programas de melhoramento genético abrangentes.

Assim, afim de elevar índices zootécnicos, otimizar o sistema de produção e atender as necessidades econômicas da venda de bovinos em diversas idades, torna-se importante conhecer informações do potencial de ganho de peso (Rezende et al., 2014) e da precocidade reprodutiva (Silveira et al., 2014). As estimativas de parâmetros genéticos para características produtivas (Toral et al., 2014; Gonçalves et al., 2011) e reprodutivas (Dias et al., 2008) em bovinos são indicadores da resposta ao melhoramento genético a ser alcançado. O conhecimento das tendências genéticas e fenotípicas de uma população é importante para se avaliar o resultado dos programas de seleção adotados, servindo como elementos norteadores de ações futuras (Passaforo et al., 2016). Objetivou-se estimar os parâmetros genéticos, e as tendências genéticas e fenotípicas para os pesos ajustados aos 205, 365, 550 dias de idade, idade ao primeiro parto, intervalos de partos e circunferência escrotal, em animais da raça Nelore criados a pasto na região Nordeste do Brasil.

\section{MATERIAIS E MÉTODOS}

Utilizaram-se informações de 175231 animais, nascidos no período de 1967 a 2011, no Nordeste Brasileiro, cedidos pela Associação Brasileira de Criadores de Zebu. As características analisadas foram: pesos ajustados aos 205 (P205/ n= 71 177), 365 (P365/n= $38583)$ e $550(\mathrm{P} 550 / \mathrm{n}=30504)$ dias de idade, idade ao primeiro parto (IPP/ $n=14231)$, circunferência escrotal $(C E / n=2970)$ aos 550 dias de idade e intervalos de partos (IDP/ n=19143). Os grupos de contemporâneos (GC) constituíram-se de animais do mesmo sexo, época (estação e ano de nascimento) e fazenda para P205 (GC= 4383), P365 (GC= 3456), P550 (GC= 2919). Para IPP $(\mathrm{GC}=1588)$ e CE $(\mathrm{GC}=161)$ aos 550 dias de idade o grupo de contemporâneos incluiu época e fazenda. Para IDP $(G C=3154)$ foi incluído a época de nascimento (parto anterior) e fazenda. Grupos de contemporâneos com menos de quatro animais foram excluídos. Para obtenção das estimativas das variâncias e dos valores genéticos, empregou-se a metodologia da máxima verossimilhança restrita livre de derivada, por meio de modelos animais unicaracterística, com uso do aplicativo MTDFREML (Boldman et al., 1995).

O modelo para P205 e P365 incluíram os efeitos aleatórios genéticos aditivos (direto e materno), efeito de ambiente permanente materno, a covariável idade da vaca ao parto (efeitos linear e quadrático), além do efeito de GC. Para P550, IPP e CE aos 550 dias de idade foi considerado apenas o efeito genético aditivo direto como efeito aleatório. Adicionalmente, ao efeito aleatório direto, foi incluído o efeito de ambiente permanente para a característica IDP. Obteve-se as estimativas das tendências genéticas e fenotípicas para as características avaliadas pela regressão linear ponderada da média da variável dependente sobre o ano de nascimento.

\section{RESULTADOS E DISCUSSÃO}

As estimativas de herdabilidade direta e materna para P205 $\left(\mathrm{h}_{\mathrm{d}}^{2}=0,15 \pm 0,0 / \mathrm{h}_{\mathrm{m}}{ }_{\mathrm{m}}=0,04 \pm 0,01\right), \mathrm{P} 365\left(\mathrm{~h}_{\mathrm{d}}{ }_{\mathrm{d}}=\right.$ $\left.0,19 \pm 0,0 / \mathrm{h}_{\mathrm{m}}^{2}=0,01 \pm 0,01\right)$ foram baixas, indicando que nessas características, a seleção fenotípica ou individual poderia acarretar baixos ganhos genéticos. As condições ambientais, podem ter exercido influência sobre características de produção e adaptação dos animais (Ferreira et al., 2014), dificultando dissimilar as variações genéticas dentro do rebanho. De acordo com Ambrosini et al. (2016), dificuldades nas identificações dos melhores animais em ambientes desfavoráveis, pode levar à equivocada utilização de reprodutores em ambientes melhores.

A estimativa de herdabilidade para P550 $\left(\mathrm{h}_{\mathrm{d}}^{2}=\right.$ $0,26 \pm 0,0)$ foi média, demonstrando possibilidade de ganho genético por meio da seleção. Já a herdabilidade direta estimada para IPP $\left(\mathrm{h}_{\mathrm{d}}^{2}=0,07 \pm 0,0\right)$ foi baixa, indicando dificuldade para o melhoramento da característica através de seleção direta. Para Laureano et al. (2011), a grande influência dos efeitos genéticos não aditivos e de ambiente na IPP indicam que a expressão da característica depende de condições adequadas de manejo. A herdabilidade direta encontrada para a característica IDP $\left(\mathrm{h}_{\mathrm{d}}^{2}=0,01 \pm 0,0\right)$ foi próxima a zero, indicando que a seleção não trará ganho genético. As diferenças nas estimativas de herdabilidades do IDP está relacionada com o manejo reprodutivo e nutricional adotado. Variações no clima e falha na obtenção do cio são exemplos comuns que ocorrem em regiões onde a criação de gado é exclusivamente a pasto.

A herdabilidade direta estimada para CE aos 550 $\left(\mathrm{h}_{\mathrm{d}}^{2}=0,37 \pm 0,0\right)$ dias de idade foi moderada. Essa característica é correlacionada favoravelmente com idade à puberdade e IPP das fêmeas (Yokoo et al., 2007) e características físicas e morfológicas do sêmen dos machos (Dias et al., 2008). Assim, podem-se obter resultados favoráveis utilizando o CE como critério de seleção. Adicionalmente, é uma característica de fácil mensuração e de baixo custo (Yokoo et al., 2007).

As tendências genéticas diretas foram significativas $(\mathrm{p}<0,0001)$ e iguais a $0,05 \mathrm{~kg} /$ ano (P205), $0,06 \mathrm{~kg} /$ ano (P365) e 0,09 kg/ano (P550), equivalendo a um ganho de $2,7 \mathrm{~kg} / \mathrm{ano} ; 2,8 \mathrm{~kg} / \mathrm{ano}$; de 4,1 kg/ano, respectivamente, durante os 45 anos avaliados. A partir de 1997 houve um maior progresso genético nas três características, indicando a existência de seleção nos rebanhos da região nestes últimos anos. Lacerda et al. (2014) estimaram ganhos de $0,06 \mathrm{~kg} / \mathrm{ano} ; 0,03 \mathrm{~kg} /$ ano e 0,04 $\mathrm{kg}$ /ano, para P205, P365 e P550, respectivamente, em bovinos da raça Nelore no estado da Bahia. Enquanto que progresso genético inferior foi relatado na raça Nelore Mocho em todo o Nordeste Brasileiro por Amaral et al. (2014), que estimaram tendências genéticas diretas de 0,01; 0,04 e 0,04 kg/ano, para P205, P365 e P550 respectivamente.

A tendência genética para a IPP foi significativa $(p<0,001)$ com coeficiente de regressão igual -0,09 dias / ano. Esse resultado pode ser explicado em razão da exposição das fêmeas em idades mais jovens na estação de monta, o que permite que alguns animais expres- 
sem seu potencial genético para precocidade sexual. Assim, a seleção que vem sendo praticada nos últimos anos está proporcionando melhorias na eficiência reprodutiva do rebanho, com a redução na idade ao primeiro parto das fêmeas.

As tendências genéticas maternas foram significativas $(\mathrm{p}<0,001)$ para P205 $(0,0039 \mathrm{~kg} / \mathrm{ano})$ e P365 $(0,0082 \mathrm{~kg} / \mathrm{ano})$. Os valores observados indicam pequeno progresso genético para o efeito materno nas duas pesagens, indicando a necessidade de mais atenção para o efeito materno nos programas de seleção, principalmente para as idades mais jovens. Essa mesma recomendação foi sugerida por Souza et al. (2011) ao verificaram em seu estudo efeito genético materno com decréscimo de $2,8 \mathrm{~kg}$ e 1,3 kg para P205 e P365, respectivamente, durante os 27 anos de estudo.

A tendência genética para a característica IDP foi significativa $(\mathrm{p}<0,001)$ e igual a 0,03 dias por ano. Esse resultado, mostra uma perda, apesar de pequena, desfavorável para a caraterística. A tendência genética para a característica CE não foi significativa, sendo um indicativo que essa característica não foi como critério de seleção no período. As tendências fenotípicas para as características P205, P365 e P550 foram significativas $(p<0,001)$ e respectivamente iguais a 1,36 kg/ano; 1,70 kg/ano e 1,90 kg/ano, representando incrementos de 47,$60 ; 59,50$ e $66,50 \mathrm{~kg}$ durante o período analisado (35 anos). As tendências fenotípicas refletem o progresso da característica na sua própria unidade de medida, possibilitando observar se os programas de seleção conjuntamente com melhorias ambientais têm sido favoráveis a produção ao longo do tempo.

Valores próximos para o progresso fenotípico, no período de 1942 a 2006, foram relatados na raça $\mathrm{Ne}$ lore na região Norte do Brasil (Malhado et al., 2010). Estes autores estimaram valores iguais a 1,17; $156 \mathrm{e}$ 1,95 kg/ano para P205, P365 e P550, respectivamente. Resultados próximos também foram relatados na raça Nelore, no estado da Bahia, por Lacerda et al. (2014), com progressos estimados de 1,13 kg/ano (P205), 1,25 kg/ano (P365) e 1,69 kg/ano (P550). Por outro lado, Amaral et al. (2014) estimaram ganhos fenotípicos superiores para P365 (2,10 kg/ano) e P550 $(2,84 \mathrm{~kg} /$ ano $)$ na raça Nelore Mocho no Nordeste.

O coeficiente de regressão para a média de idade ao primeiro parto foi significativo $(\mathrm{p}<0,001)$ e igual a -7,06 dias por ano. Malhado et al. (2010) encontraram um coeficiente de regressão para a média de idade ao primeiro parto de $-33,4$ dias por ano, estudando a raça Nelore na região Norte. A tendência fenotípica para a característica CE aos 550 dias de idade foi significativa $(p<0,035)$, com um ganho de 0,24 $\mathrm{cm} /$ ano, o que corresponde a um incremento de 2,1 $\mathrm{cm}$ durante o período de 09 anos estudados. Visto que a tendência genética para CE aos 550 dias de idade não foi significativa, o progresso fenotípico observado pode ser devido a uma forte contribuição genética não aditiva e em decorrência de melhorias nas condições ambientais.

\section{CONCLUSÃO}

As estimativas dos coeficientes de herdabilidades para as características de crescimento foram de baixa a moderada magnitude. A seleção direta proporcionará maiores ganhos genéticos para P550. Para as características reprodutivas, as estimativas de herdabilidades foram baixas para IPP e próxima a zero para IDP. Contudo, para a CE aos 550 dias de idade a herdabilidade foi moderada, indicando seu possível uso como critério de seleção. As tendências genéticas diretas para P205, P365, P550 e IPP, indicam pequenos ganhos genéticos ao longo dos anos. As características estão melhorando com o decorrer dos anos, principalmente em decorrência da melhoria ambiental.

\section{BIBLIOGRAFIA}

Amaral, R.S.; Carneiro, P.L.S.; Martins Filho, R.; Ambrosini, D.P. e Malhado, C.H.M. 2014. Tendências, parâmetros fenotípicos e genéticos de características de crescimento em bovinos Nelore mocho do Nordeste brasileiro. Rev Bras Saúde Prod Anim, 15: 261-271.

Ambrosini, D.P.; Malhado, C.H.M.; Martins Filho, R. e Carneiro, P.L.S. 2016. Interação genótipo $x$ ambiente via modelos de normas de reação para características de crescimento em bovinos Nelore. Pesq Agropec Bras, 51: 177-186.

Biffani, S.; Martins Filho, R.; Giogetti, A.; Bozzi, R. e Lima, F.A.M. 1999. Environmental and genetic effects on yearling and post-yearling weights of Nellore calves in Northeast Brazil. Rev Bras Zootecn, 28: 468-473.

Boldman, K.G.; Kriese, L.A.; Van Vleck, L.D., Van Tassel, C.P.V. eKachman, S.D. 1995. A manual for use of mtdfreml; a set of programs to obtain estimates of variances and covariances [DRAFT]. Department of Agriculture, Agricultural Research Service. Lincoln. 120 pp.

Dias, J.C.; Andrade, V.J.; Martins, J.A.M.; Emerick, L.L. e Vale Filho, V.R. 2008. Genetic and phenotypic correlations among reproductive and productive traits of Nellore bulls. Pesq Agropec Bras, 43: 53-59.

Ferreira, J.L.; Lopes, F.B.; Assis, A.S. e Lobo, R.B. 2014. Climatic spatialization and analyses of longitudinal data of beef cattle Nellore raising Maranhão, Pará and Tocantins using univariate and multivariate approach. Semin Cienc Agrar, 35: 2197-2210.

Fialho, F.R.L.; Rezende, M.P.G.; Souza, J.C.; Silva, R.M.; Oliveira, N.M. e Silveira, M.V. 2015. Performance in preweaning pure and crossbred calves in the Mato Grosso do Sul Pantanal region, Aquidauana, Mato Grosso do Sul State, Brazil. Acta Sci, Anim Sci, 37: 437-442.

Gonçalves, F.M.; Pires, A.V.; Pereira, I.G.; Garcia, D.A.; Farah, M.M.; Meira, C.T. e Cruz, V.A.R. 2011 . Avaliação genética para peso corporal em um rebanho Nelore. Arq Bras Med Vet Zootec, 63: 594-598.

Lacerda, J.J.D.; Carneiro, P.L.S.; Martins Filho, R. eMalhado, C.H.M. 2014. Parâmetros e tendências genéticas para características de crescimento em bovinos da raça Nelore no Estado da Bahia utilizando inferência bayesiana. Rev Bras Saúde Prod Anim, 15: 10-19.

Laureano, M.M.M.; Boligon, A.A.; Costa, R.B.; Forni, S.; Severo, J.L.P. e Albuquqerque, L.G. 2011 . Estimativas de herdabilidade e tendências genéticas para características de crescimento e reprodutivas em bovinos da raça Nelore. Arq Bras Med Vet Zootec, 63: 143-152.

Malhado, C.H.M.; Carneiro, P.L.S. e James, R.P. 2010. Melhoramento genético e estrutura populacional da raça Nelore no Norte do Brasil. Pesq Agropec Bras, 45: $1109-1116$.

Marques, E.G.; Magnabosco, C.U.; Lopes, F.B. e Silva, M.C. 2013. Estimativas de parâmetros genéticos de carcateristicas de crescimento, carcaça e perímetro escrotal de animais da raça Nelore avaliados em provas de ganho de peso em confinamento. Biosc J, 29: 159-167.

Oliveira, N.M.; Rezende, M.P.G.; Abreu, U.G.P.; Rosa, A.N.; Sterza, F.A.M. e Fialho, A.L.L. 2015. Environmental effects on reproductive performance of Nellore cows widely raised in the Cerrado/Pantanal ecotone. Acta Sci, Anim Sci, 37: 77-82. 
Passaforo, T.L.; Fragomeni, B.O.; Gonçalves, D.R.; Moraes, M.M. e Toral, F.L.B. 2016. Genetic analysis of body weight in a Nellore cattle herd. Pesq Agropec Bras, 51: 149-158.

Rezende, M.P.G.; Silveira, M.V.; Silva, R.M.; Silva, L.O.C.; Gondo, A.; Ramires, G.G. e Souza, J.C. 2014. Pre and post weaning weight gain in Nellore cattle raised in the Pantanal, Mato Grosso do Sul, Brazil). Cienc Anim, 24: 20-27.

Silveira, M.V.; Souza, J.C.; Silva, L.O.C.; Freitas, J.A.; Gondo, A.B. e Ferraz Filho, P.B. 2014. Interação genótipo x ambiente sobre características produtivas e reprodutivas de fêmeas Nelore. Arch Zootec, 63: 223-226.
Souza, J.C.; Silva, L.O.C.; Gondo, A.; Freitas, J.A.; Malhado, C.H.M.; Filho, P.B.F.; Sereno, J.R.B.; Weaber, R.L. e Lamberson, W.R. 2011. Parâmetros e tendência genética de peso de bovinos criados á pasto no Brasil. Arch Zootec, 60: 457-465.

Toral, F.L.B.; Pereira, J.C.C.; Bergmann, J.A.G. e Josahkian, L.A. 2014. Parâmetros genéticos do peso desde o nascimento até 730 dias de idade na raça Indubrasil. Pesq Agropec Bras, 49: 595-603.

Yokoo, M.J.I.; Albuquerque, L.G. de; Lôbo, R.B.; Ssainz, R.D.; Júnior, J.M.C.; Bezerra, L.A.F. e Araujo, F.RC. 2007. Estimativas de parâmetros genéticos para altura do posterior, peso e circunferência escrotal em bovinos da raça Nelore. Rev Bras Zootecn, 36: 1761-1768. 